\title{
Conocimientos y practicas sobre el consumo de tabaco en estudiantes de pregrado de farmacia, Lima, Perú
}

\author{
Delia Danjoy León ${ }^{1}$ \\ Paulo Sérgio Ferreira ${ }^{2}$ \\ Sandra Cristina Pillon ${ }^{3}$
}

El objetivo del estudio fue determinar los conocimientos y prácticas del consumo de tabaco en estudiantes de farmacia, según aspectos demográficos y académicos. Participaron 276 (55.2\%) estudiantes de farmacia de una universidad privada Peruana. Fue utilizada la Encuesta Global de Tabaco (GYTS) para jóvenes. Se identificó una prevalencia de vida del tabaco de $93,7 \%$ en los hombres y en las mujeres de 77,6\%. La prevalencia de vida para el alcohol fue de $70,8 \%$ y para las drogas ilegales de 14,1\% (marihuana). Existe un elevado porcentaje de prevalencia de vida y de consumo actual de tabaco en esta muestra. La mayoría de los estudiantes inicio el uso a los 16 años. Existe una fuerte asociación entre el consumo de tabaco y el uso de esta droga por, al menos, uno de los padres; también está asociado a la exposición al humo dentro de la casa.

Descriptores: Conocimiento; Práctica Asociada; Tabaquismo; Estudiantes; Farmacia.

\footnotetext{
${ }^{1}$ Profesor Auxiliar, Facultad de Enfermería, Universidad Peruana Cayetano Heredia, Perú. E-mail: ddanjoy@upch.edu.pe.

2 Enfermero, Escola de Enfermagem de Ribeirão Preto, Universidade de São Paulo, Centro Colaborador de la OMS para el Desarrollo de la Investigación en Enfermería, SP, Brasil. E-mail: pausefer@eerp.usp.br.

${ }^{3}$ Enfermeira, Doutor, Professor Doutor, Escola de Enfermagem de Ribeirão Preto, Universidade de São Paulo, Centro Colaborador da OMS para o Desenvolvimento da Pesquisa em Enfermagem, Brasil. E-mail: pillon@eerp.usp.br.
}

Correspondencia:

Sandra Cristina Pillon

Universidade de São Paulo. Escola de Enfermagem de Ribeirão Preto

Av. Bandeirantes, 3900

Bairro Monte Alegre

CEP: 14040-902 Ribeirão Preto, SP, Brasil

E-mail: pillon@eerp.usp.br 


\section{Conhecimentos e práticas sobre o consumo de tabaco entre estudantes de farmácia em Lima, Peru}

O objetivo do estudo foi determinar os conhecimentos e as práticas do uso de tabaco entre estudantes de farmácia, segundo os aspectos sociodemográficos e acadêmicos. Participaram $276(55,2 \%)$ estudantes de farmácia de uma universidade privada peruana. Foi utilizada a Encuesta Global de Tabaco para jovens - GYTS. Identificou-se a prevalência do uso na vida de tabaco em $93,7 \%$ dos homens e $77,6 \%$ das mulheres. A idade de início do consumo ocorreu aos 16 anos. A prevalência de uso na vida para o álcool foi de $70,8 \%$ e, para as drogas ilegais, $14,1 \%$ para a maconha. Existe porcentagem elevada de estudantes que fizeram uso na vida e, ainda, consomem tabaco, nesta amostra. A maioria dos estudantes iniciou o uso de drogas aos 16 anos. Existe forte associação entre o consumo de tabaco entre os que possuem pelo menos um dos pais fumantes e a exposição ao fumo dentro de casa.

Descritores: Conhecimento; Prática Associada; Tabagismo; Estudantes; Farmácia.

\section{Knowledge and Practice Regarding Tobacco Use Among Pharmacy Undergraduate Students in Lima, Peru}

The aim of this study was to determine the knowledge and the practices regarding tobacco consumption in pharmacy students according to their demographic and academic characteristics. The sample consisted of 276 (55.2\%) pharmacy students of a Peruvian Private University to whom the "Global Youth Tobacco Survey" (GYTS) was applied. In men, the prevalence of lifetime use of tobacco of $93.7 \%$, of alcohol $70.8 \%$ and of illegal drugs $14.1 \%$ was identified. The prevalence of lifetime and current consumption of tobacco in the sample was very high. The majority of students began using drugs at 16 years old. There was a strong association between the consumption of tobacco and the fact that at least one of the parents smoked and the exposure to smoke inside the home.

Descriptors: Knowledge; Partnership Pratice; Smoking; Students; Pharmacy.

\section{Introducción}

El tabaco a través de la historia pasó por momentos de gran aceptación social y alcanzó proporciones muy grandes, no solo por el número de fumadores en el planeta, sino también por las graves consecuencias que trae para la salud y para los costos sociales. En ese sentido, parece que fue lento el proceso de reconocimiento, por parte de los hombres, de los daños provocados por la nicotina y sus consecuencias. En un estudio de revisión(1) se presentan dos informes mundiales realizados por la Organización Mundial de la Salud (OMS) por dos investigadores sobre el tema, ellos informan que cada año el número de fumadores nuevos se incrementa, mucho más en los países de baja economía; se estima que diariamente surgen entre 64 mil y 84 mil nuevos fumadores, mientras que en los países de economía alta esa proporción es bastante menor, situándose entre 14 y 15 mil. En esa revisión el autor ${ }^{(1)}$ comenta también que, en el mundo, cerca de 1,2 billones de personas son fumadoras, produciéndose casi 4 millones de muertes por enfermedades asociadas al tabaquismo. En los Estados Unidos el tabaquismo provoca 400 mil muertes al año, número que es mayor que la suma de las muertes provocadas por el alcohol y todas las demás drogas licitas e ilícitas; muriendo anualmente 50 mil fumadores pasivos, es decir, aquellos que no fuman pero que inhalan el humo del tabaco de otros fumadores.

Mediante estas evidencias no se puede ignorar el daño que produce el tabaquismo para los intereses de los profesionales del área de la salud, pues es un tema 
de alta prioridad para las acciones de salud pública que no solo está dirigido a los especialistas sino también a todos los profesionales de la salud que deben estar preparados y capacitados para abordar esa problemática junto a sus clientes. Los currículos de los estudiantes, en formación, de las profesiones de salud poco abordan el tema de drogas, hecho que contribuye para el estereotipo negativo y los sentimientos inadecuados de los diversos profesionales para lidiar con este problema. El uso del tabaco entre los profesionales de la salud, principalmente cuando todavía son universitarios es bastante discutido en la literatura(2-5).

Los comportamientos de fumary las actitudes de los profesionales de la salud han sido ampliamente discutidos por el papel que desempeñan como profesionales responsables ${ }^{(4-5)}$, por otro lado, los profesionales de la salud pueden contribuir decisivamente en la reducción del uso de tabaco, mediante abordajes terapéuticos simples y consejos a la población afectada, lo que incrementaría sustancialmente el promedio de cesación de fumar. En ese contexto, los profesionales de la salud están en una posición privilegiada para influenciar el comportamiento de otras personas en la decisión de parar de fumar.

Los índices del hábito tabáquico entre los estudiantes y profesionales de la salud son altos y preocupantes en toda América Latina ${ }^{(1-2,6)}$.

Un estudio realizado en Ecuador(2) que incluyó una muestra de estudiantes de pregrado del primer año, identificó que el $65 \%$ ya había fumado cigarrillos por lo menos una vez y en relación al uso actual, el $20 \%$ eran fumadores, ejerciendo el consumo en la propia universidad.

En el Perú, un estudio(4) sobre el uso de tabaco y otras drogas entre estudiantes del área de ciencias de la salud identificó el consumo de tabaco en 75,8\%, de alcohol en 94,5\%, marihuana 4,8\%, PBC 0,6\% y clorhidrato de cocaína $1,2 \%$. Además, en ese país otro estudio realizado entre estudiantes de enfermería de 18 Facultades/Escuelas pertenecientes a la Asociación Peruana de Facultades y Escuelas de Enfermería encontró que el 71,8\% consumía tabaco, siendo que el conocimiento sobre tabaco y otras drogas fue bajo, con reducida participación en las actividades promotoras de salud y estilos de vida saludables(5).

En Brasil, diversas universidades vienen evaluando y monitoreando el comportamiento de fumar y de beber entre los estudiantes, con el interés de ofrecer acciones preventivas a esa población, una vez que éstos se inician en el consumo, principalmente de alcohol y de tabaco, en la etapa de estudiantes(3). Otro estudio realizado en el mismo país entre estudiantes de farmacia identificó que el $40 \%$ ya fumaba ${ }^{(6)}$.

En Bolivia, también se identificó que la mitad de los estudiantes bolivianos ha fumado(7). Entre los estudiantes fumadores, resaltaron los de género masculino y en relación al conocimiento, es mayor la proporción de personas fumadoras entre las que no tienen conocimientos de los daños causados por el tabaco a la salud (73\%) que entre las personas con ese conocimiento (50\%).

Los estudiantes que fuman y no practican deportes o ejercicios físicos de manera regular alcanzan el 51\%, porcentaje mayor que la proporción de personas que fuman y practican deporte o ejercicio físico en forma regular (42\%). La proporción de personas de género masculino que fuma (49\%) es superior a la proporción de personas de género femenino que fuma (39\%). La proporción de personas con hábito de beber (85\%) es mayor entre fumadores que entre no fumadores $(47.5 \%)$. Más de la mitad de los estudiantes que beben 206 (56\%) lo hacen por cuestiones sociales y empezaron a fumar porque les atrajo la idea de hacerlo $131(49 \%)$ y por la influencia de los amigos $60(22 \%)^{(7)}$.

Con respecto a los conocimientos de los estudiantes universitarios sobre el tabaquismo, realizado entre estudiantes españoles, el 42,5\% considera el alquitrán como el componente más tóxico del humo del tabaco. Asociaron con el hábito tabáquico al cáncer de pulmón $(97,9 \%)$. El incremento del riesgo de complicaciones durante el embarazo es la afirmación con la que está totalmente de acuerdo el 74,5\%. El 15,4\% entienden el lema "Tabaco o Salud, elija". Reconoce que los profesionales sanitarios influyen en los hábitos de la población el $62,7 \%{ }^{(8)}$.

Frente a las estadísticas del uso de tabaco, las actitudes de los estudiantes y la escasez de conocimientos, específicamente entre los estudiantes de farmacia, el presente estudio tuvo como objetivo identificar sus conocimientos y el uso de tabaco entre estudiantes del pregrado de farmacia en una Facultad de Farmacia en Lima, Perú.

\section{Método}

Es un estudio del tipo transversal, realizado en la Facultad de Farmacia de una Universidad de Lima, Perú. 
Participaron 278 (55,2\%) estudiantes de pregrado. Los datos fueron obtenidos por encuesta voluntaria autoaplicada, mediante firma del Consentimiento Informado; el estudio fue aprobado por el Comité de Ética de la Universidad Peruana Cayetano Heredia. El instrumento utilizado fue una versión adaptada del Global Youth Tobacco Survey (GYTS)(9), con 92 preguntas cerradas de opción múltiple y selección única. Con confiabilidad para esta muestra fue de 0,877 (alfa de Cronbach). Para el análisis estadístico se empleó el programa Statistical Package Social Science for Windows v.10. Se emplearon las pruebas de Chi-cuadrado $\left(X^{2}\right)$ y de Odds Ratio (OR) con Intervalo de confiabilidad (IC) de $95 \%(p<0,05)$ considerados significativos.

\section{Resultados}

La Tabla 1 muestra que 64\% de los estudiantes han fumado alguna vez en la vida, de los cuales el 93,7\% son de género masculino, y $96,6 \%$ se encuentran en edades entre 27 y 30 años y, el 95,8\% se encontraba cursando el 5 año de estudios.

Tabla 1 - Prevalencia de vida de consumo de tabaco en estudiantes de farmacia según aspectos sociodemográficos y académicos. $(n=278)$ Lima, Perú. 2007

\begin{tabular}{lcc}
\hline & Fuma & No Fuma \\
\cline { 2 - 3 } & $\%$ & $\%$ \\
\hline Género & \\
Masculino & 93,7 & 6,3 \\
Femenino & 77,6 & 22,4 \\
Edad & & \\
16 a 18 años & 70,2 & 29,8 \\
19 a 22 años & 86,5 & 13,5 \\
23 a 26 años & 80,7 & 19,3 \\
27 a 30 años & 96,6 & 3,4 \\
31 a más años & 71,4 & 28,6 \\
Año del curso & & \\
$1^{\circ}$ año & 72,7 & 27,3 \\
$2^{\circ}$ año & 80,5 & 19,5 \\
$3^{\circ}$ año & 89,2 & 10,8 \\
$4^{\circ}$ año & 87,8 & 12,2 \\
$5^{\circ}$ año & 95,8 & 4,2 \\
\hline
\end{tabular}

La Tabla 2 demuestra que en relación al consumo actual de tabaco, 54,4\% pertenecen al género masculino y $32,2 \%$ al género femenino; $45,6 \%$ se encuentran entre las edades de 23 y 26 años, y por último, mitad de los fumadores actuales está en el quinto año de estudios.
Tabla 2 - Condición actual de consumo de tabaco de estudiantes de farmacia según aspectos sociodemográficos y académicos. $(n=278)$ Lima, Perú. 2007

\begin{tabular}{lccc}
\hline & $\begin{array}{c}\text { Fumador } \\
\text { actual }\end{array}$ & Ex-fumador & $\begin{array}{c}\text { Nunca ha } \\
\text { fumado }\end{array}$ \\
\cline { 2 - 4 } & $\%$ & $\%$ & $\%$ \\
\hline Género & 54,4 & 39,2 & 6,3 \\
Masculino & 32,2 & 45,4 & 22,4 \\
Femenino & 87,5 & 8,3 & 4,2 \\
No responde & & & \\
Edad & 42,6 & 27,7 & 29,8 \\
16 a 18 años & 38,1 & 48,4 & 13,5 \\
19 a 22 años & 45,6 & 35,1 & 19,3 \\
23 a 26 años & 44,8 & 51,7 & 3,4 \\
27 a 30 años & 28,6 & 42,9 & 28,6 \\
31 o más años & & & \\
Año de estudio & 36,4 & 36,4 & 27,3 \\
$1^{\circ}$ año & 39,0 & 41,5 & 19,5 \\
$2^{\circ}$ año & 49,2 & 40,0 & 10,8 \\
$3^{\circ}$ año & 46,9 & 40,8 & 12,2 \\
$4^{\circ}$ año & 50,0 & 45,8 & 4,2 \\
$5^{\circ}$ año & 50,0 & 50,0 & 0,0 \\
No responde & 43,3 & 40,4 & 16,2 \\
\hline Total & & & \\
\hline
\end{tabular}

La Figura 1 muestra que los estudiantes de ambos géneros se inician en el consumo de tabaco a los 16 o más años de edad.

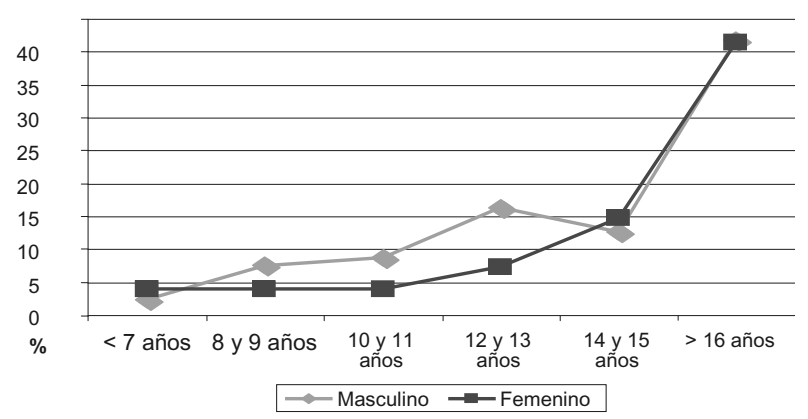

Figura 1 - Edad de inicio de consumo de tabaco en estudiantes de Farmacia. Lima, Perú. 2007

Una cuarta parte de los estudiantes refieren que nunca han fumado y los que han fumado refieren que el lugar donde fuman más frecuentemente es en los eventos sociales $(22,8 \%)$ y en el centro de estudios $(12,9 \%)$.

En relación al uso de otras drogas, el alcohol fue el de mayor uso, 196 (70,8\%) de los estudiantes manifestaron haber consumido alcohol alguna vez en la 
vida. En relación a la marihuana $(14,1 \%)$ fue la droga mas usada, seguido por lo PBC $33(11,9 \%)$ y $27(9,7 \%)$ por la cocaína.

Acerca de la exposición al humo de tabaco, la mitad de los estudiantes de farmacia estuvo expuesta al humo del tabaco alguna vez en la semana, dentro de la casa $(41,9 \%)$ y más de la mitad se expone fuera de casa $(57,5 \%)$.

Al respecto de las políticas restrictivas del fumar, $203(73,3 \%)$ de los estudiantes están de acuerdo en que se prohíba fumar en lugares públicos.

La Tabla 3 muestra los factores de riesgo asociados al fumar, existe una fuerte asociación entre el uso de tabaco y la presencia de que, al menos, uno de los padres sea fumador; también está asociado con la exposición dentro de casa y con el consumo de drogas ilegales como marihuana y PBC.

Tabla 3 - Factores de riesgo asociados al consumo de tabaco entre estudiantes de farmacia $(n=278)$ Lima, Perú. 2007

\begin{tabular}{lcccc}
\hline & $\chi^{2}$ & p. & \multicolumn{2}{c}{ OR (IC 95\%) } \\
\hline Tus padres fuman & 4,701 & $0,030^{*}$ & 2,267 & $(1,067 ; 4,817)$ \\
Tus hermanos fuman & 2,240 & 0,134 & 2,977 & $(0,670 ; 13,238)$ \\
Exposición dentro de casa & 11,943 & $0,001^{*}$ & 3,690 & $(1,698 ; 8,019)$ \\
Exposición fuera de casa & 2,968 & 0,085 & 1,752 & $(0,921 ; 3,333)$ \\
Es difícil dejar de fumar & 0,178 & 0,673 & 1,200 & $(0,515 ; 2,798)$ \\
una vez que uno ha & & & & \\
empezado & & & & \\
Consumo de Marihuana & 9,295 & $0,002^{*}$ & 5,536 & $(1,643 ; 18,649)$ \\
Consumo de PBC & 5,605 & $0,018^{*}$ & 7,954 & $(1,057 ; 59,849)$ \\
Consumo de Alcohol & 0,084 & 0,772 & 0,859 & $(0,308 ; 2,395)$ \\
\hline
\end{tabular}

*valores de $p<0,05$ foram consideradas significativos

\section{Discusión}

La mayoría de los estudios realizados entre estudiantes universitarios, principalmente los relacionados a las profesiones de la salud, demuestran que la prevalencia de vida de tabaco es alta y preocupante cuando lo comparamos con la población general(2-5,7).

De acuerdo con las características sociodemográficas y académicas de los 278 (100\%) estudiantes del presente estudio, fue predominante el género femenino 175 (62,9\%), con edad entre 19 y 22 años, 126 (45,5\%) del segundo año de estudios $(29,6 \%)$, más de la mitad solamente se dedican a estudiar y residen en la zona norte de la ciudad de Lima, semejante a los estudios de poblaciones estudiantiles ${ }^{(2-5)}$.

La prevalencia de vida del tabaco entre los estudiantes de farmacia fue de 232 (83\%), mientras que
45 (16,2\%) nunca habían fumado; estos porcentajes son mayores a los encontrados en otros estudios realizados en estudiantes de las profesiones de la salud(10), y de una universidad privada de $\operatorname{Lima}^{(4)}$ y en la población nacional urbana Peruana(11). Además es muy superior a la encontrada en estudios internacionales ${ }^{(4,6)}$.

Al analizar la prevalencia de vida según el género, existe un predominio entre estudiantes fumadores de género masculino $(93,7 \%)$, las estudiantes femeninas fueron el $77,6 \%$, esta característica de predominancia fue similar al estudio realizado en Lima(4). De acuerdo con la historia de uso de tabaco(1), éste se diseminó primero en el género masculino y mucho mas tardíamente en el género femenino, el autor resalta que la importancia epidemiológica del tabaquismo en la mujer se acentuó a partir de la Segunda Guerra Mundial.

En relación a la edad, el consumo es mayor en el grupo de 27 a 30 años y en estudiantes de 5to año. Resultados similares se muestran en otro estudio, en el cual se observó que conforme aumenta la edad de los estudiantes, llegan al final del período de estudio y la condición de género masculino, se incrementan la probabilidad de consumo de tabaco(12).

El hecho de encontrar en el estudio diferencias entre la prevalencia de vida y uso actual en los estudiantes de género masculino y femenino, nos debe llevar a planificar acciones de prevención y control y líneas de investigación con un enfoque de género, a fin de tener un conocimiento más claro sobre las nuevas tendencias de consumo.

Al analizar el consumo actual de tabaco, encontramos un $43,3 \%$, cifra superior a la de otros estudios $^{(4,12)}$, los que muestran índices cada vez mayores de consumo de tabaco en estudiantes de nivel superior, con una tendencia ascendente al consumo. Se analizó la prevalencia general de consumo desde el año 1989 hasta 1996, encontrándose que aumentó, la variación fue $11 \%$ en 1989 y de $28,8 \%$ en 1996 .

El uso actual presenta las mismas características que la prevalencia de vida, la cual es mayor en los estudiantes de género masculino $(54,4 \%)$, con edad entre 23 a 26 años $(45,6 \%$ ) y mitad se encuentran en el 5to año de estudios

Al indagar sobre la edad de inicio, Figura 1, la mayoría de los estudiantes de farmacia, de ambos géneros $(41,8 \%)$ se inicia en el consumo de tabaco a los 16 o más años de edad. Este dato nos lleva a pensar que el fumar en los estudiantes está empezando antes del ingreso a la Universidad; esto sumado a la edad de inicio y a la presencia de uno de los miembros de 
la familia que fuma, lo que puede contribuir directa o indirectamente como modelo y ser factores reforzadores para su uso(2). Otro estudio reporta que la edad de inicio también se caracteriza por que se produce en la adolescencia temprana, período conflictivo, en que generalmente las personas intentan fumar por primera vez ${ }^{(12)}$. Por otro lado, son personas que no alcanzan la edad legal para el consumo de tabaco. En ese sentido, la literatura enfatiza que es preocupante el incremento observado en el consumo de tabaco en edades inferiores al mínimo legalmente permitido para poder adquirir tabaco. También, se informa que además del problema de salud, los datos del estudio ponen de manifiesto otras fallas, como el no cumplimiento de las regulaciones de venta de tabaco(12). Situación similar podemos encontrar en Perú y otros países, donde a pesar de los avances en la Ley de Prevención y Control de uso indebido del tabaco, se puede observar la libre venta de cigarrillos al por menor, por vendedores ambulantes y con ausencia de control por parte de las autoridades.

Al indagar sobre el conocimiento que tienen los estudiantes de farmacia sobre las drogas se identificó que apenas la mitad posee conocimientos regulares sobre las drogas y solo uno de cada diez posee buenos conocimientos. Sin embargo más de la mitad manifestó conocer que el humo de cigarrillo que fuman otras personas les hace daño.

Frente a la escasez de conocimientos específicos de los estudiantes con respecto al tabaquismo y otras drogas, se identifica la necesidad de contar con un currículo de estudios que aborde esta temática, conteniendo una mayor investigación y exploración de las actitudes de los futuros profesionales de la salud y con énfasis en la prevención y control del uso de drogas.

En cuanto a la peligrosidad de las sustancias, los estudiantes opinan que las sustancias ilegales como éxtasis, PBC, cocaína y marihuana son las sustancias más peligrosas $y$, en menor proporción indicaron peligrosidad en las legales (tabaco y alcohol). Esto nos lleva a pensar sobre el desconocimiento sobre los efectos del tabaco sobre la salud de las personas. La literatura $^{(1)}$ evidencia más de 50 causas de morbilidad y mortalidad que son mucho más comunes en fumadores, siendo el tabaquismo un factor de alto riesgo, asociado a 25 de ellas.

Los estudiantes respondieron que es en eventos sociales (53\%), donde acostumbran a fumar con mayor frecuencia, pero $30 \%$ fuman en el centro de estudios. El centro de estudios es un local de interacción social para los estudiantes y las políticas de control para no fumar son casi inexistentes. Estudios al respecto son escasos, esto demuestra la falta de una política más efectiva para el control del tabaquismo en las universidades. Un estudio realizado en el Ecuador muestra que el uso del cigarrillo es diferente que el del alcohol, éste no puede ser vendido en la universidad, sin embargo no es prohibido su consumo, esto demuestra la permisividad social en relación al tabaco(2).

Entre las drogas legales, el alcohol presenta una prevalencia de vida, entre los estudiantes de farmacia de $70,8 \%$, porcentaje alto, pero concuerda con las cifras de la población urbana Peruana $(87,2 \%)$; para el uso en la vida del alcohol se encuentra proporciones similares entre el género(11) y las de estudiantes de ciencias de la salud $(94,3 \%)^{(6)}$. La literatura evidencia que el uso de alcohol se tornó un habito tradicional entre esa población, elegido como la sustancia más consumida entre estudiantes ${ }^{(2-6)}$.

También, esos índices son preocupantes, más todavía si los encontramos en poblaciones en edades que corresponden a la adolescencia y juventud. Los autores refieren que los estudiantes no perciben el riesgo de usar drogas ilegales, solo el 52\% de los estudiantes opinó que el uso del crack es más arriesgado y el 51,5\% el uso de cocaína(6)

Los comportamientos de fumar y las actitudes de los profesionales de la salud han sido ampliamente discutidos en el contexto de su papel como profesionales responsables ${ }^{(4-5)}$, sin embargo, los profesionales de la salud pueden contribuir decisivamente en la reducción del uso de tabaco, mediante abordajes terapéuticos simples y aconsejando a la población afectada, lo que incrementaría sustancialmente el promedio de cesación de fumar. En ese contexto, los profesionales de la salud están en una posición privilegiada para influir en la decisión de otras personas para no fumar.

Los índices del hábito tabáquico entre los estudiantes y profesionales de la salud son altos y preocupantes en toda Latinoamérica, como muestran los estudios ${ }^{(2-6)}$.

Frente a los resultados de este estudio, donde se identificó una prevalencia de vida y una prevalencia actual elevada del consumo de tabaco entre los estudiantes, los cuales se convertirán en futuros profesionales; se ha diagnosticado la necesidad inmediata de realizar intervenciones por parte de las autoridades universitarias a fin de: fortalecer los contenidos de este tema del tabaco y otras drogas en el currículo de estudios; hacer reflexionar a los estudiantes sobre el papel que les compete desarrollar como futuros profesionales de la salud; producir más investigaciones que permitan, no 
solo conocer el diagnóstico de la situación de consumo, sino también analizar las tendencias y los factores de riesgo asociados; evaluar la efectividad de los programas de intervención, y otros que permitan revertir la situación encontrada y analizada en el presente estudio.

\section{Conclusiones}

Ese trabajo buscó identificar la prevalencia de vida y el uso actual del tabaco, así como los conocimientos y opinión sobre el uso de sustancias psicoactivas entre los estudiantes de farmacia de una universidad privada Peruana. Esta es la prima vez que se evalúa el uso de tabaco entre estudiantes de farmacia. En vista de los altos índices de uso, este estudio nos muestra indicadores para desarrollar trabajos preventivos en el ámbito universitario, además de la necesidad de realizar mayores inversiones en los contenidos curriculares sobre el tema de drogas, dada la importancia que tiene la futura formación de los profesionales de la salud, especialmente la de farmacéuticos, con la finalidad de garantizar una actuación profesional que sea sensible ante la importancia del comportamiento de fumar y de las actitudes frente a esa temática, que debe considerar como un problema de la salud pública.

\section{Agradecimientos}

Agradecemos a la Comisión Interamericana para el Control del Abuso de Drogas/CICAD de la Secretaria de Seguridad Multidimensional/SSM de la Organización de los Estados Americanos/OEA, la Secretaria Nacional de Políticas sobre Drogas/SENAD do Gabinete de Seguridad Institucional/Brasil, la Escuela de Enfermería de Ribeirao Preto de la Universidad de Sao Paulo y Centro Colaborador de la Organización Mundial de la Salud para el Desarrollo de la Investigación en Enfermería, la población representada en los estudios de investigación, bien como a las autoridades de las universidades representadas por los participantes del Programa En-Line de Especialización en Investigación sobre el Fenómeno de las Drogas PREINVEST, periodos 2005, 2006, 2007 y 2008.

\section{Referencias}

1. Rosemberg J, Miranda MA, Rosemberg AMA. Nicotina - Droga Universal. São Paulo (BR): Instituto Nacional de Câncer (INCA); 2004. [citado em 27 jul 2008]. Disponível em: www.inca.gov.br/ tabagismo/publicacoes/nicotina.pdf.

2. Piedra KAC, O'Brien B, Pillon SC. Drugs use and risk behavior in a university community. Rev. Latino-Am. Enfermagem. 2005 novembro-dezembro; 13(número especial):1194-200.

3. Pillon SC, O'Brien B, Piedra KAC. The relationship between drugs use and risk behaviours in brazilian university students. Rev. Latino-Am. Enfermagem. 2005 novembro-dezembro; 13(número especial):1169-76.

4. Zárate M, Zavaleta A, Danjoy D, Chanamè E, Prochazka R, Salas M, Maldonado V. Prácticas de Consumo de Tabaco y otras drogas en estudiantes de ciencias de la salud de una universidad privada de Lima, Perú. Invest Educ Enferm 2006; 24(2):72-81. 5. Zárate $M$, Zavaleta $A$, Chanamè E. Conocimientos y Prácticas de Consumo de Tabaco y otras drogas en Estudiantes de 18 facultades / escuelas peruanas de Enfermería, 2003. Rev Peruana Enferm, Investigación y Desarrollo 2005; 7(1):50-60. 6. Oliveira TB, Azeredo F, Prado D, Rezende A, Cunha LC, Garrote CFD. Uso de álcool, tabaco e drogas ilícitas por estudantes de farmácia da UFGO. Rev Eletrônica Farmácia 2005; 2(2):133-6.
7. Rosas RB. Incidencia del Hábito de Fumar y tomar bebidas alcohólicas en la población estudiantil de UNIVALLE. Cochabamba. Bolivia; Junio 2003.

8. García López JA. Conocimientos de los estudiantes de Farmacia sobre el tabaquismo. Ars Pharmaceutica. 1996; 37(1):43-52.

9. Global Youth Tobacco Survey Collaborative Group. Tobacco use among youth: a cross country comparison. Tob Control 2002; 11(3):252-70.

10. Encuesta Mundial de Profesionales de la Salud (GHPS): El uso de tabaco en estudiantes de tercer año de Medicina, Enfermería y Farmacia. Perú. 2006. Centro de Información y Educación para la Prevención del Abuso de Drogas. Centro para la Prevención y Control de Enfermedades. Organización Panamericana de la Salud. Organización. Mundial de la Salud. Lima. Perú; 2007.

11. Rojas M, Castro de la Mata R. Epidemiología de las drogas en la población urbana peruana, 1997. Monografía de Investigación no 17. Centro de Información y Educación para la prevención del abuso de drogas. CEDRO Lima, Perú;1999.

12 Valdés SR, Micher JM, Hernandez L, Hernandez M, HernandezAvila M. Tendencias de consumo de tabaco entre alumnos de nuevo ingreso a la Universidad Autónoma de México, 1989 a 1998. Salud Pública Mex. 2002; (44): supl 1:44-S53. 